Background and aims Skin to Skin (STS) care in neonatal period influences immediate breastfeeding outcomes in early childhood, especially the duration of exclusive breastfeeding. We investigated influence of STS care given on day one of life on infant and child feeding (IYCF) practices through one year of life.

Methods Mothers of 100 neonates (48 girls, 52 boys) from previous study cohort of RCT on STS care was followed. A telephonic survey on IYCF practices during the first year of life was administered.

Results There was no difference in the groups as far as the duration of exclusive breastfeeding, number of times breastfed per day, or stoppage of night feeds. No baby in either group received bottled feeds but about 53 received some form of extra lacteal feeds in the first 6 months without significant group difference. Fewer STS group mothers reported difficulties with breastfeeding or extra lacteal supplementation. All mothers who faced problems contacted physicians for advice, and 20 were advised top milk and 6 given other foods. At one year of life $66 \%$ mothers were giving less than the recommended five food servings. There was no difference in practices related to handwashing, food preparation and storage, feeding habits of child and illness episodes in the children.

Conclusions IYCF practices even in this small group were not as per recommended guidelines. Few positive trends were seen with fewer STS mothers facing problems related to breastfeeding. The study was underpowered to detect differences in IYCF practices in relation to STS care.

\section{P0-0129 ALPHA-1 ANTITRYPSIN: CHARACTERISATION OF CHILDREN FOLLOWED IN A LEVEL 2 HOSPITAL}

K Oliveira, S Oliveira, D Soares, I Nunes, C Rocha, L Gomes, M Costa. Pediatria, Centro Hospitalar de Entre Douro E Vouga, Santa Maria Da Feira, Portugal

10.1136/archdischild-2014-307384.793

Background Deficiency of Alpha-1 Antitrypsin (D-AAT) is one of the most common serious hereditary diseases worldwide, however, remains an under diagnosed entity.

Objectives To characterise children with D-AAT followed in a level 2 hospital (2000-2013).

Methods Retrospective analysis of clinical records of children with D-AAT followed in a paediatrics digestive pathology consultation.

Results 41 cases (61\%males) were diagnosed. The average age at diagnosis was 6 yrs $\left(1\right.$ month $^{-1} 5$ yrs).

The diagnosis was established in the following settings: study of allergies in $26.83 \%$; family history in $21.95 \%$; lower respiratory infections in $9.76 \%$; asymptomatic elevation of transaminase in $7.32 \%$; Escreva texto ou o endereço de um Web site ou traduza um documentoTraduzirdo: Portuguêsneonatalcholestasis, prolonged neonatal jaundice, and persistent transaminase elevations after acute gastroenteritis in $4.88 \%$ each; others pathologies in $34.14 \%$.

Immunophenotyping allowed detection of alleles $\mathrm{Z}$ and $\mathrm{S}$, in $85 \%$ and $40 \%$, respectively. Other pathological alleles identified less frequently were the I, V and Mmaltom. The phenotype identified were: $\mathrm{MZ}$ in $32.5 \%$, SZ $26.8 \%, \mathrm{ZZ} 19.5 \%$, SS and IZ4.88\% each; MS, MV, SV, and SM maltom $2.5 \%$ each.

In $100 \%$ of cases the presence of at least one disease in parental allele was verified.

Discussion The most common pathological allele was the $\mathrm{Z}$, and the predominant phenotype was MZ. All children are clinically well, without complications. We highlight the need for awareness of the scientific community for the early diagnosis of this entity in order to implement interventions to prevent the progression of lung disease by decreasing any proinflammatory stimuli, and to undertake family studies to ensure early diagnosis of other cases and provide genetic advice.

\section{PO-0130 CAMPYLOBACTER GASTROENTERITIS IN CHILDREN}

${ }^{1} \mathrm{~K}$ Oliveira, ${ }^{1} \mathrm{~B}$ Aguiar, ${ }^{1} \mathrm{I}$ Nunes, ${ }^{2} \mathrm{~A}$ Pedrosa, ${ }^{2} \mathrm{H}$ Costa, ${ }^{1} \mathrm{~L}$ Gomes, ${ }^{1} \mathrm{M}$ Costa. ${ }^{1}$ Pediatrics, Centro Hospitalar de Entre Douro E Vouga, Feira, Portugal; ${ }^{2}$ Clinical Pathology, Centro Hospitalar de Entre Douro E Vouga, Feira, Portugal

10.1136/archdischild-2014-307384.794

Introduction Acute gastroenteritis (AGE) remains, throughout Europe, a public health issue. Campylobacter is the most common enteropathogen after the age of five, particularly in Northern Europe.

Objective Epidemiological data of the paediatric population with AGE by Campylobacter.

Methods Retrospective data collection from clinical records of patients less than 18 years with confirmed Campylobacter positive stool cultures over a one year period.

Results Out of 322 patients with AGE and at least one or more clinical criteria for stool culture (fever, blood or mucus stools in our case), in 83 (25.8\%) Campylobacter was isolated. Although, this was the most frequently identified agent, there was, an inverse relationship with increasing age (mean age 2.8 years; range 3 weeks to 17 years). Most cases (39.8\%) occurred in the spring. Serotypes isolated were C. jejuni in $83.1 \%$ and C. coli in $3.6 \%$. Possible food items responsible included free range home produced eggs and non-potable water. In 12\% there was a family history of illness and in one patient theres was a previous infection by Campylobacter. Eleven (13.3\%) children required admission. Antibiotics were started prior to stool culture results in only two cases; following the stool cultures only one child was given antibiotics, having the remaining $98.8 \%$ clinically improved by then.

Discussion AGE is a self-limited disease with most patients recovering within a weak. Rehydration and electrolyte correction is the mainstay of treatment.

\section{PO-0131 VALUE OF STOOL CULTURE OF CHILDREN}

${ }^{1} \mathrm{~K}$ Oliveira, ${ }^{1} \mathrm{~B}$ Aguiar, ${ }^{1} \mathrm{I}$ Nunes, ${ }^{2} \mathrm{~A}$ Afonso, ${ }^{2} \mathrm{~F}$ Silva, ${ }^{1} \mathrm{~L}$ Gomes, ${ }^{1} \mathrm{M}$ Costa. ${ }^{1}$ Pediatrics, Centro Hospitalar de Entre Douro e Vouga, Feira, Portugal; ${ }^{2}$ Clinical Pathology, Centro Hospitalar de Entre Douro e Vouga, Feira, Portugal

\subsection{6/archdischild-2014-307384.795}

Introduction Acute gastroenteritis (AGE) remains, throughout Europe, a public health issue. Under the age of 5, some 20 to $30 \%$ of bacterial microorganisms are identified. However, costeffectiveness of routine stool cultures yielding only $2 \%$ results preclude routine stool culturing.

Objective Evaluation of the value of stool culture of children with AGE.

Methods Retrospective data collection from clinical records of patients less than 18 years old submitted to stool cultures over a one year period.

Results Out of 322 stool culture, 56.8\% fulfilled the accepted ESPGHAN criteria and $74.8 \%$ had at least 1 clinical predictor of positivity (fever, blood or mucus stools, $>10$ bowel actions/24 $\mathrm{h}$, abdominal pain, travelling to highly epidemic countries). 